\title{
Osteogenic-related gene expression by human adipose-derived mesenchymal stem cells
}

\author{
I. Khlusov ${ }^{1,2}$, L. Litvinova ${ }^{2 *}$, K. Yurova ${ }^{2}$, V. Shupletsova ${ }^{2}$, O. Khaziakhmatova ${ }^{2}$, \\ E. Melashchenko², L. Pokrovskaya ${ }^{1}$ \\ ${ }^{1}$ Tomsk State University, Tomsk, Russia \\ ${ }^{2}$ Immanuel Kant Baltic Federal University, Kaliningrad, Russia \\ *e-mail: larisalitvinova@yandex.ru
}

Key words: in vitro, quantitative polymerase chain reaction, osteoblastic differentiation

Motivation and Aim: Mesenchymal stem cells (MSCs) are multipotent cells, which among other cell lineages, give rise to adipocytes and osteoblasts. The differentiation of adipose-derived MSCs (AMSCs) into osteoblasts occurs through the cross talk between complex signaling pathways including those derived from bone morphogenic proteins (BMPs), fibroblastic growth factors (FGF), osterix (OSX), and runt-related transcription factor 2 (Runx2). Transcription factors Runx2 and OSX are the main molecular switches and determinants of MSC osteoblastogenesis [1]. Therefore, an expression of osteogenic genes has been studied for 14-day culture of human AMSCs (hAMSCs).

Methods and Algorithms: hAMMSCs were isolated from lipoaspirate (permission no. 7 of December 9, 2015; Local Ethics Committee, Immanuel Kant Baltic Federal University) as described in [2]. Cells were cultured with standard nutrient medium ( $90 \%$ DMEM/F12 (1:1), $10 \%$ fetal-calf serum, $50 \mathrm{mg} / \mathrm{mL}$ gentamicin, and $280 \mathrm{mg} / \mathrm{mL}$ L-glutamine without osteogenic additions) for 14 days with medium exchange every 3-4 days. The expression of osteogenic-related genes (RUNX2, BMP2, BMP6, FGF10, and $A L P L$ ) was analyzed via multiplex quantitative polymerase chain reaction (qPCR). mRNA was extracted from cells using the ExtractRNAkit (Evrogen, Russia) according to the manufacturer's instructions. Reverse transcription of RNA samples was performed using the MMLV RT kit (Evrogen, Russia). qPCRmixHS reagents (Evrogen, Russia), specific TaqMan probes and primers (Beagle, Russia) were used for qPCR with the help of CFX96 amplifier (Bio-Rad, USA). RPLPO served as reference gene.

Results: The expression of pro-osteogenic genes was determined in 14 days of hAMMSCs culturing. Testing of corresponding transcription proteins in particular Runx2 and OSX is planning.

Conclusion: Isolated hAMSCs are useful cells for in vitro genetic investigation of osteoinductive properties of scaffolds proposed for bone tissue engineering.

Acknowledgements: Supported by the Federal Target Program of the Ministry of Education and Science of the Russian Federation (Agreement No. 14.575.21.0164, ID number RFMEFI57517X0164).

\section{References}

1. Muruganandan S. et al. (2008) Adipocyte differentiation of bone marrow-derived mesenchymal stem cells: Cross talk with the osteoblastogenic program. Cell. Mol. Life Sci. 66(2):236-253.

2. Zuk P.A. et al. (2011) Multilineage cells from human adipose tissue: implications for cell-based therapies. Tissue Engineering. 7(2):211-228. 